\title{
CME
}

\section{Anatomy of aging face}

\section{Rakesh Khazanchi, Aditya Aggarwal, Manoj Johar ${ }^{1}$}

Department of Plastic and Cosmetic Surgery, Sir Ganga Ram Hospital, New Delhi - 110 060, ${ }^{1}$ Fortis Hospital, Noida, UP, India

Address for correspondence: Dr. Rakesh Khazanchi, Department of Plastic and Cosmetic Surgery, Sir Ganga Ram Hospital, New Delhi - 110 060, India. E-mail: rkkhazanchi@hotmail.com

ejuvenation of the face is evolving into a common procedure in India. This may be attempted by either surgical or non surgical means. Surgical rejuvenation of face includes a large variety of procedures to revert the changes of aging. In the past, face lift operation was done to simply lift the sagging skin rather than shaping the face. However it often ended up in giving the patient an 'operated on' look producing tight appearing face. The surgeons have now learnt that aging process is a complex process that involves soft tissues as well as skeleton of face and is not just sagging of skin. Therefore in order to get a good result after surgical facial rejuvenation, it is paramount to understand these anatomical structures and the effect of aging process on them. The proportion in which these changes occur in different individuals also varies and the surgical procedure needs to be designed according to specific needs of each patient. Surgical rejuvenation of face has evolved from a simple face lift operation to a combination of procedures such as:
a) Excision of skin and subcutaneous of tissues
b) Tightening of lax structures
c) Remodeling of skin with lasers, mesotherapy
d) Building of tissues with fat or synthetic fillers
e) Building up of bony deficiencies

\section{Why do changes occur with aging?}

This happens due to a variety of reasons. These are

a) Constant effect of gravity on soft tissue results in their sagging over the facial skeleton

b) Sun damage to skin

c) Hormonal changes in women around menopause

d) Decreased skin blood flow associated with aging

e) Weight gain due to slow down of metabolism and fat deposition in regions of body called 'depots'

f) Fascial and ligament laxity

g) Shrinkage of glandular tissue (Salivary glands)

h) Skeletal resorption

Facial soft tissues are arranged in concentric layers. Skin is the outermost layer and then the basic building blocks-fat, superficial fascia also known as superficial musculoaponeurotic system (SMAS), deep fascia and the periosteum that covers the facial skeleton. Interspersed in these layers are vessels, nerves, facial muscles and retaining ligaments. Knowledge of these layers allows the surgeon to dissect in a given anatomic plane without damaging important structures. Knowledge of the changes that occur with aging allows the surgeon to make changes such as excision, tightening, addition or remodeling in order to restore youthful appearance to face. This article reviews the basic anatomy of the face in a simple manner and covers each layer individually along with the changes that occur with aging.

Skin: Skin is the investing layer of facial soft tissues and skeleton. Youthful skin is smooth, firm, uniform in colour, texture and without creases and wrinkles. Histologically skin consists of epidermis and dermis. Dermis is composed primarily of collagen, elastic fibers and ground substance. It can be divided into superficial papillary dermis and deeper reticular dermis. The papillary layer consists of collagen bundles, elastic fibers, fibrocystic and ground substances and has highly developed microcirculation. The ground substance found in papillary dermis is composed of mucopolysaccharides, hyaluronic acid and chondroitin sulphate. The reticular dermis is composed of thick bundles of coarse collagen with interspersed elastic fibers. Ground substance tends to have a gel like consistency 
giving a youthful look to the skin. Cutaneous aging is a complex process consisting of two components; intrinsic aging which is genetically determined and extrinsic aging caused by environmental exposure, primarily U-V light.

Sun induced actinic damage or solar elastosis is the most important reason of earliest skin changes. These changes may appear as early as mid-20s but are usually apparent by mid-30s. Smoking further contributes to skin damage. The dermis begins to loose collagen and elastin. Ground substance begins to decrease and get replaced by fibrous tissue. By the fourth decade of life, the rate of cell renewal of decreases. This causes the epidermis to become loose and skin creases become evident in a pattern that is perpendicular to the direction of muscle action. In women, the estrogen levels decrease at menopause, which causes further thinning of the dermis, irregularity of the epidermis and atrophy of fat. Blood supply to skin decreases and causes loosening of the epidermis and rough texture of skin. There is decrease in sebaceous gland activity leading to dry skin. Hyperpigmentation is another prominent feature of aging skin. It occurs because of increased melanin in the epidermis, the dermis or both.

Textural changes of the skin start with fine superficial lines in the skin - wrinkles. With time the lines increase and get organized. Those due to facial muscle contractions, known as MIMETIC LINES get added. With repeated muscular contractions these mimetic lines extend into the dermis causing SKIN FURROWS and later, when skin becomes redundant and over lapping, SKIN FOLDS become apparent [Figure 1].

Wrinklesover theface which are commonly seen are 'worry lines' - transverse lines in the forehead perpendicular to frontalis; 'frown lines'- oblique lines in the glabellar region perpendicular to corrugator; 'Crow's feet' - lateral periorbital lines perpendicular to orbicularis occuli; 'smile line or the nasolabial' line perpendicular to zygomaticus muscle and lines from corner of mouth to jowls is known as 'marionette lines'. Other commonly seen lines on the face are cheek lines, chin lines, commissural lines and transverse neck lines and are all perpendicular to the underlying muscles.

The net effects of all these changes on skin are dullness, irregular texture, irregular pigmentation, dryness, thinning, mimetic lines, furrows, folds and sagging.

Clinically, it is important to differentiate these mimetic lines and furrows from skin folds. Mimetic lines and furrows respond well to multi modality therapy such as injectable fillers, botulinum toxin and or laser resurfacing. Skin folds on the other hand require surgical tightening procedures like brow lift, blepharoplasty and face lift etc. Since mimetic lines, furrows and folds are all present simultaneously in patients seeking facial rejuvenation, a combination of surgical and non surgical methods is required in most patients to get optimal results.

Fat: Fat, along-with superficial fascia, forms the basis of facial volume and contour. This fact is obvious in patients suffering from facial lipodystrophy [Figure 2].

Subcutaneous fat in the face is divided into superficial and deep layers by the superficial fascia which in the face is called Superficial Musculo Aponeurotic System (SMAS).

Superficial fat is a continuous layer of fat that is not uniform. It is arranged as small yellow lobules interwoven with fibrous septa that connect SMAS to dermis. This superficial fat is densest in the cheek, nasolabial fold, jaws, anterior neck and pre mental areas. It is sparse in the forehead, temporal orbital, perioral and posterior neck triangle regions [Figure 3].

The deep layer of fat is below the SMAS. It is discontinuous and arranged as large white lo bules with sparse network of thin fibrous septa. It is most dense in temporal, periocular, anterior and middle cheek and submental region. In temporal region the deep fat consists of temporal fat pad and the deep temporal extension of fat pad known as fat pad of Bichat. In the periocular region the fat pad deep to the orbital septum is called 'periorbital fat pad' and that deep to the orbicularis oculi pad is known as suborbicularis oculi fat pad (SOOF) [Figure 4].

With aging, changes that occur in the two layers of the facial fat are different. There is loss of superficial subcutaneous fat due to decreased vascularity. On the other hand, there is increased accumulation of fat in the deep fat layer, the so called 'depots'. This is due to slowing down of metabolism after the $4^{\text {th }}$ decade of life. In women, decreasing estrogen level after the age of 50 further contributes to increased fat in deep depots and paradoxical loss of superficial subcutaneous fat.

\section{Superficial fascia (superficial musculo aponeurotic system -SMAS)}

The superficial fascia in the face is called the SMAS. In the 
past SMAS was thought to be a fibromuscular network between the subdermal fat and the facial nerve branches, located above the platysma musculature and below the superficial temporal musculature. However recent

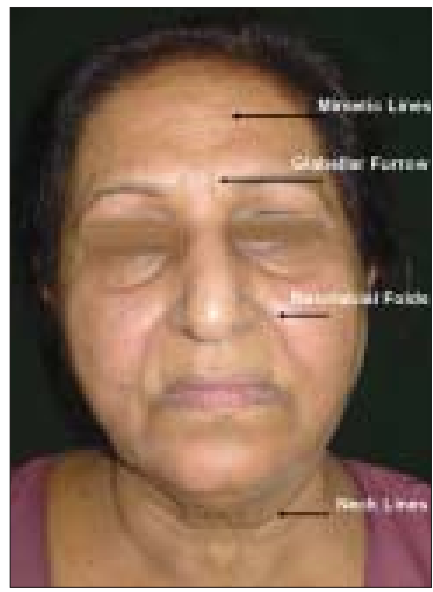

Figure 1: Textural changes in skin in a post menopausal patient showing mimetic lines, wrinkles, furrows and folds

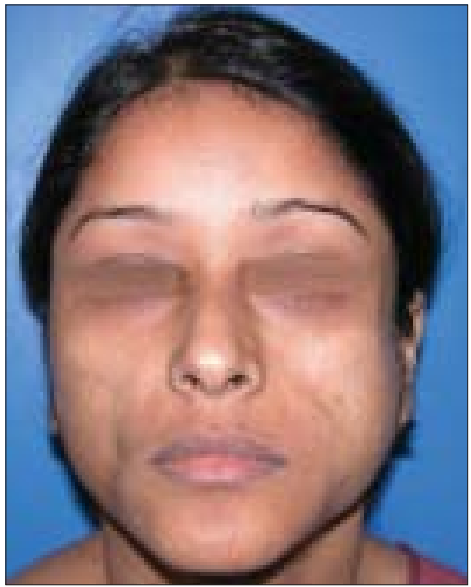

Figure 2: Loss of facial contours seen in a patient with facial lipodystrophy

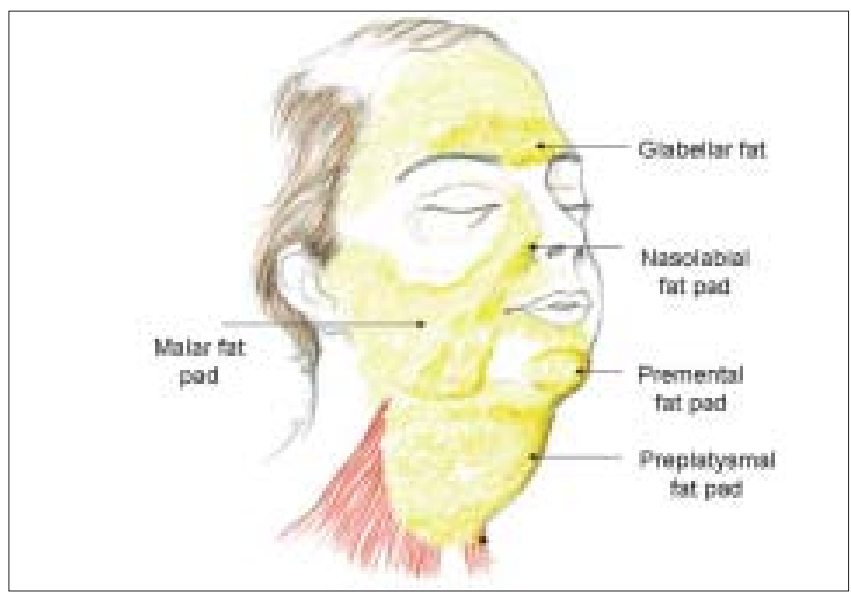

Figure 3: Superficial fat distribution research has shown SMAS to be composed of several layers that split to invest facial muscles. Facial nerve lies deep to SMAS in its lateral part and pierces its deep layers in its central part to innervate the facial muscles. SMAS is

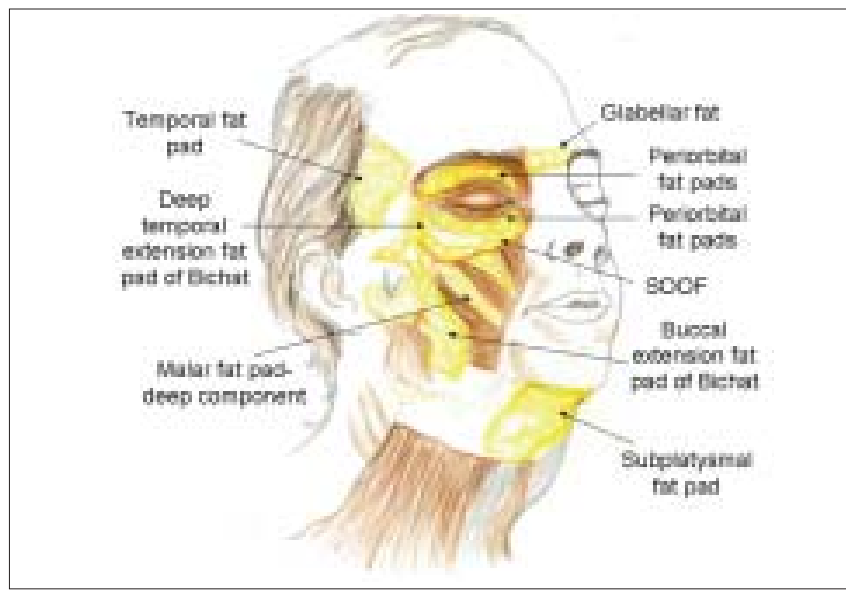

Figure 4: Deep fat distribution

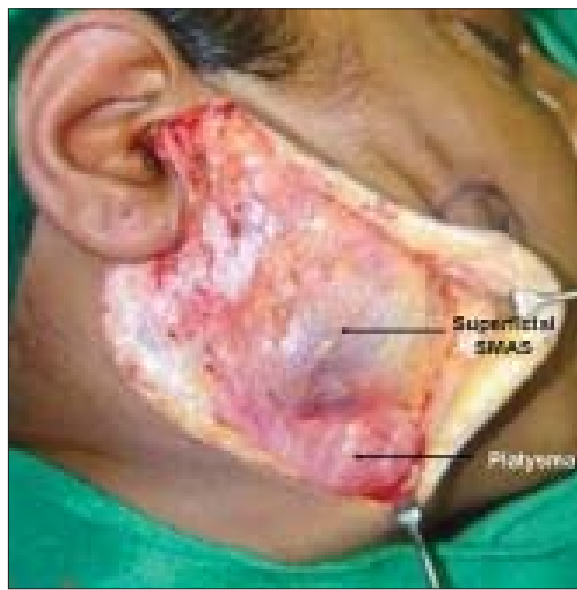

Figure 5: SMAS as seen after lifting the skin flap with superficial fat in patient undergoing surgery for a vascular malformation

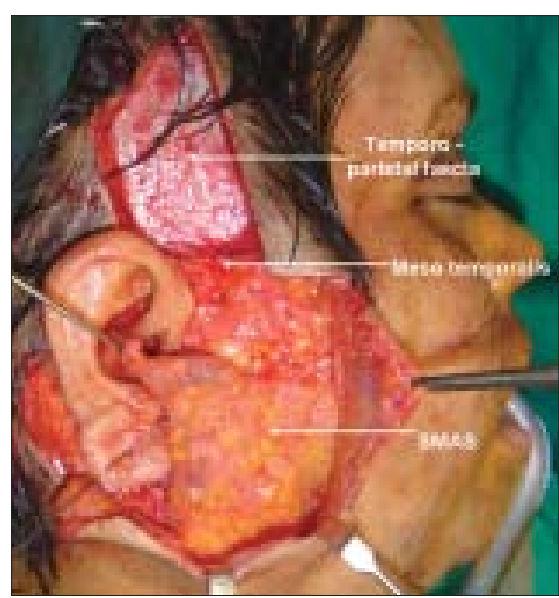

Figure 6: SMAS and superficial temporal fascia following elevation of skin flap in a patient undergoing face lift 
the most important structure that is used to lift the facial skin, fat, platysma and muscles since it is attached to all these structures [Figure 5].

In the region of parotid gland and masseter muscle, SMAS is thick and uniform [Figure 6]. It is densely adherent to the capsule of parotid gland. It thins out in the anterior cheek region. In this region, the SMAS forms facial mimetic and platysma musculature. It actually splits to invest these muscles in between these two layers

In the temporoparietal region the superficial fascia is called temporoparietal fascia and in the scalp it is called galea. The frontal branch of facial nerve pierces the deep layer of temporo parietal fascia after it crosses the mid portion of zygomatic arch. It then travels within this fascia to innervate the forehead muscles.

Deep facialfascia: Onlifting theSM ASand temporoparietal fascia the deep facial fascia can be seen [Figure 7]. In the temporal region it is called the deep temporal fascia and is made of two layers-superficial and deep.

The temporal fat pad is situated between the superficial and deep layers of deep temporal fascia. Deep to the deep layer is the temporalis muscle and in the same plane just above the zygomatic arch is the temporal component of fat pad of Bichat also known as the deep temporal fat pad [Figure 4].

Below the zygomatic arch, the deep fascia is a thin translucent layer covering the masseter muscle, facial nerve, facial artery, vein and parotid duct. This is the parotido masseteric fascia. More anteriorly, the buccal fat pad of Bichat lies deep to this fascia. The parotido massetric fascia is thin and fragile. Great care must be taken while dissecting the SMAS off the thin layer, particularly as one proceeds more anteriorly as the facial nerve branches pierce this fascia to innervate the superficial facial mimetic muscles. In the upper mid face a pad of fat is seen deep to orbicularis Oculi-known as the sub orbicularis oculi fat (SOOF). The SOOF is responsible for malar bags which are situated inferolateral to lower eyelid bags.

Following the dissection of deep facial fascia (deep temporal fascia and parotido masseteric fascia), the deep facial structures become evident. These are the temporalis muscle and deep temporal fat pad above the zygomatic arch, masseter muscle, buccal fat pad, deep facial mimetic muscles (levator anguli oris, buccinator and mentalis), branches of facial nerve, facial artery and vein and parotid duct. The facial nerve branches lie superficial to branches of facial artery and vein, parotid duct and buccal fat pad and they innervate the deep facial mimetic muscles from their superficial surface [Figure 8].

The retaining ligaments: These are aponeurotic condensations of fibrous tissue that run from deeper structures to overlying dermis and help to anchor the skin and mobile soft tissues to the underlying skeleton. There are two types of retaining ligaments- true and false. True retaining ligaments are short and stout fibrous bands that run from periosteum to dermis. They are found in four locations- orbital, zygomatic, mandibular and buccal maxillary [Figure 9].

The orbital ligaments are located over zygomaticofrontal suture. The zygomatic ligaments attach the fat to the underlying zygomatic eminence. The mandibular ligaments attach the parasymphyseal dermis to the underlying bone and help support the chin pad to the underlying bone. The buccal maxillary ligaments have both true and false components. The true components attach the skin to zygomatico maxillary suture.

The false retaining ligaments fix the superficial fascia (SMAS) to the deep fascia and function like sheets of Velcro to prevent gravitational descent. They are located in three regions and are accordingly named platysma auricular ligaments, masseteric ligaments and buccal maxillary ligaments [Figure 9].

The platysma-auricular ligaments form a thick fascial aponeurosis that attaches the postero- superior border of platysma to the lobule of ear. They provide a dissection plane in the subcutaneous preauricular region that leads directly to external surface of platysma. Tissues of medial cheek are supported by a series of fibrous bands that extend between the skin and anterior border of masseter called the masseteric ligaments. When SMAS is elevated anterior to the parotido masseteric fascia, one enters a loose areolar tissue plane that leads to masseteric ligaments. Laxity of these ligaments leads to the characteristic submalar hollow in the aging face.

The soft tissues of the anterior cheek are supported by buccal maxillary ligaments situated adjacent to the nasolabial folds. They are weakest of retaining ligaments making the anterior cheek more susceptible to sagging with aging. 
Retaining ligaments are taut in youth keeping the mobile superficial face tissues firmly anchored to underlying skeleton or deep fascia. Years of muscular activity and gravity result in fascial and ligamentous laxity which in combination with dermal elastosis and increased fat in

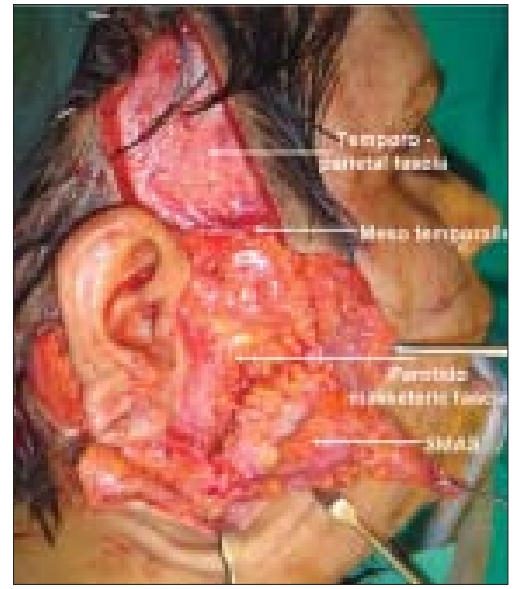

Figure 7: Parotido-masseteric fascia seen after lifting the SMAS flap

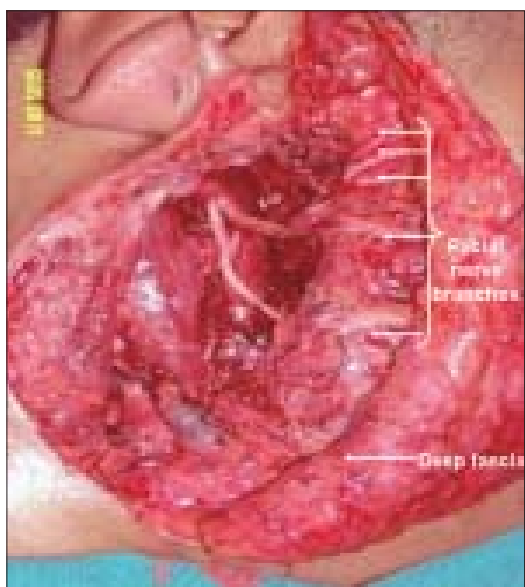

Figure 8: Facial nerve and vessels seen over the masseter muscle after lifting of skin, SMAS and deep fascia and removal of superficial lobe of parotid gland

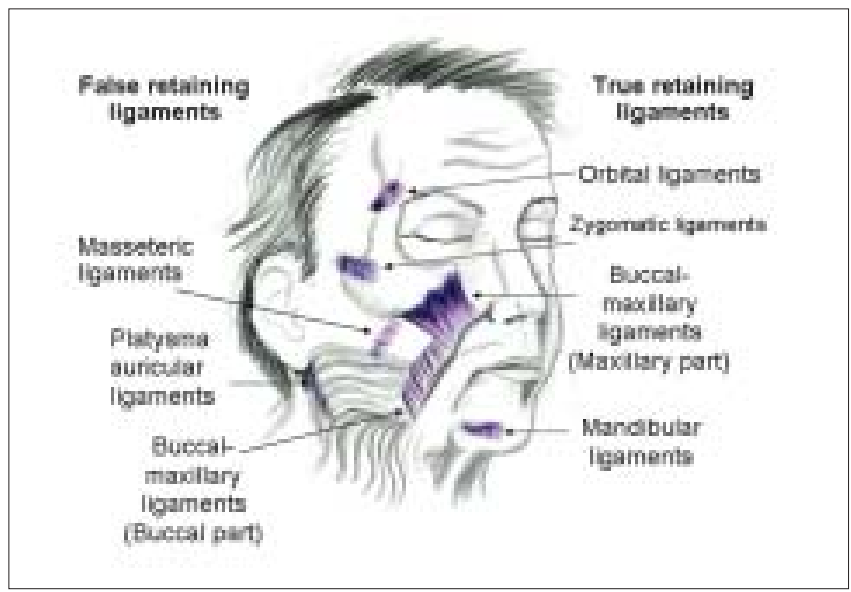

Figure 9: True and False retaining ligaments deep compartment results in descent of all elements of soft tissues.

The malar soft tissues are suspended from the zygomatic eminence and maxilla by the zygomatic ligaments laterally and buccal maxillary ligaments medially. With aging, as these ligaments become lax, there is inferior migration of malar tissues. This soft tissue ptosis occurs adjacent to line of fixation along the nasolabial fold. This leads to the prominent nasolabial fold with aging. The attenuation of masseteric ligaments leads to descent of cheek soft tissues below the mandibular border and formation of jowls. The anterior border of jowls is formed by mandibular ligaments and posterior border by masseteric ligaments.

\section{THE FACIAL NERVE}

Injury to one of the branches of the facial nerve is the most feared complication of surgical facial rejuvenation. However a thorough knowledge of anatomy makes such

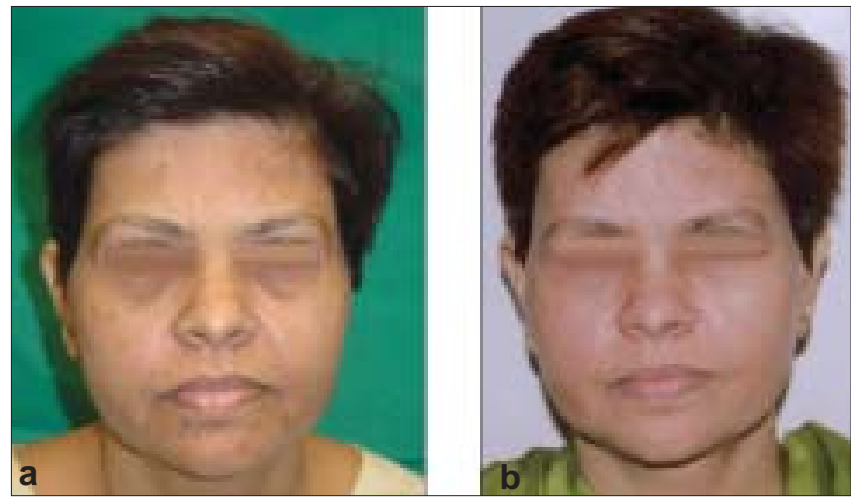

Figure 10: (a-b) Pre and 6 months post operative pictures of a 50 years old woman requesting facial rejuvenation. She was treated by a comprehensive face lift, followed by cream programme and IPL photo rejuvenation

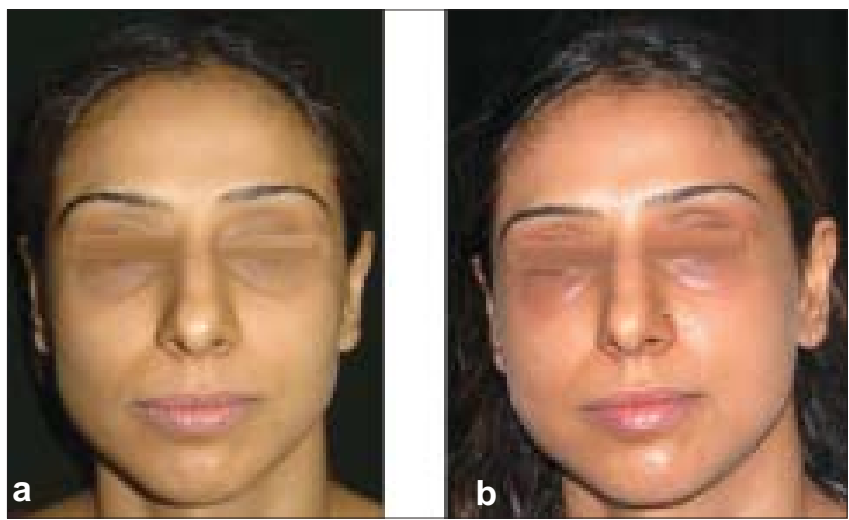

Figure 11: (a-b) Pre and 4 months post operative pictures of a 35 year old woman requesting facial rejuvenation following loss of facial contours due to weight loss. She was treated by fat injections 
an eventuality very rare. The facial nerve travels within the substance of parotid gland where it bifurcates into upper temporal and lower cervical divisions. These divisions exit the parotid gland to lie on the superficial surface of masseter muscle deep to the parotidomasseteric fascia [Figure 8]. The divisions then branch further as they pass over the masseter muscle. Anterior to the masseter muscle, the branches pierce the deep layer of SMAS to innervate the facial muscles.

The temporal division divides into five to seven branches to supply muscles in frontal orbital, zygomatic and buccal region. All branches except the frontal have many interconnections amongst themselves. Frontal branch is also the smallest and being a terminal branch, its injury causes significant motor deficit in the frontal region. It crosses the zygomatic arch at its midpoint and then pierces the deep layer of temporoparietal fascia. The key to save this nerve from injury is to dissect deep to the temporoparietal fascia in the temporal region above the zygo matic arch and superficial to parotidomasseteric fascia below the zygomatic arch, thus creating a mesentery in the zygomatic arch area called the meso-temporalis. Because of interconnections amongst the rest of the branches of upper division, any injury to one or two of these branches is unlikely to result in any facial motor deficit.

The cervical division is the smaller of two and divides into three to five branches- to buccal, mandibular and cervical regions. Like the frontal branch of the upper division, the marginal mandibular branch of the lower division is a terminal branch and is most liable to injury. Its injury causes obvious clinical deficit due to paralysis of depressor labii inferioris muscle. It enters the platysma muscle along its deep surface and is liable to injury in operations requiring elevation and division of platysma in the upper neck. Marginal mandibular nerve descends inferior to mandible in the region posterior to the facial artery lying 1-2 cm inferior to its lower border. In older individuals and with extension of neck it may lie even lower. In 10-15\% of patients there are connections between this nerve and the buccal branches. In these patients considerable recovery of function will take place even if this nerve is transected.

\section{THE FACIAL MUSCLES}

An intimate knowledge of facial muscles is essential to surgeons performing facial rejuvenation procedures. There are numerous facial muscles, which are sandwiched between two layers of facial SMAS. The SMAS has connections with the dermis through fibrous strands. The facial muscles working in groups through these fibrous strands cause multiple and complex facial movements resulting in a variety of facial expressions. Repeated action of these muscles on the skin, results in mimetic wrinkles and furrows. Grossly the facial muscles are divided into two groups- the periocular and the perioral muscles.

Of the periocular muscles, the frontalis muscle is responsible for transverse forehead lines, the corrugator supercilii are responsible for vertical glabellar furrows and procerus muscle causes transverse lines at the root of nose and glabella. The frontalis muscle is elevator of the brow and the orbicularis oculi antagonises its action and is depressor of brow. Along with corrugator supercilii, it is responsible for brow ptosis.

The perioral muscles are responsible for the movement of upper and lower lips and the angles of mouth. They are situated in two planes- superficial and deep. The SMAS splits to invest the superficial muscles (zygomaticus major and minor, platysma, risorius, depressor anguli oris and superficial head of orbicularis oris). The facial nerve branches innervate them along their deep surface. The zygomaticus major and minor which are the predominant muscles of smile can be injured during face lift surgery. The deep perioral muscles (levator labii superioris, levator anguli oris, buccinator, mentalis, depressor labii inferioris and deep portion or orbicularis oris are innervated by branches of facial nerve along their superficial surface. The buccal fat pad is situated deep to buccinators. This accounts for safety of facial muscles and nerves during excision of buccal fat pad through the buccal mucosa. The lip elevators are responsible for the naso labial crease and lip depressors (depressor anguli oris) for labio mandibular crease.

\section{BLOOD SUPPLY}

Skin of the face has a rich arterial supply through a network of fasciocutaneous perforating arteries. The peripheral zone of face is supplied predominantly by branches of superficial temporal artery (transverse facial, zygomatic-orbital and frontal). The central zone is supplied predominantly by branches of facial artery along with supraorbital and infraorbital arteries and submental artery. In commonly performed face lift operation, the lifting of preauricular and neck skin flap results in division of contributions from the branches of superficial temporal 
artery. The flap thus raised is supplied by branches of facial artery which ensures the flap survival provided it is not too thin. The facial artery is situated under the deep fascia and should never be ligated. In patients who are smokers or have had facial artery ligation in a previous surgery, the flap undermining should be conservative.

\section{NERVE SUPPLY}

The sensory nerve supply of the face is by branches of the three divisions of trigeminal nerve and by the branches of cervical plexus. Raising of the face lift flap results in its denervation. However the rich networks of sensory nerves regenerate to a completely new network within a few months. The delay in sensory recovery is most commonly seen in the lateral cheek. This region is a watershed zone between the trigeminal nerve and cervical plexus nerve territories. Sensory return in this region therefore takes the longest.

Knowledge of anatomy of greater auricular nerve is important as it can get injured during face lift surgery. It is located $6.5 \mathrm{~cm}$ inferior to the external auditory meatus crossing the sternocleidomastoid muscle. It is located deep to the superficial cervical fascia which is a continuation of SMAS in the neck. As long as the subcutaneous undermining of the lateral neck flap does not violate this fascia, this nerve is safe. If injured, this nerve should be repaired to avoid loss of sensation to the lobule and posterior auricle.

\section{CONCLUSIONS}

The facial soft tissues are made of concentric layers with skin outside, skeleton on the inside and fat, muscles and fascia in between. The retaining ligaments fix the facial soft tissues to each other and underlying skeleton. With aging, changes occur in all these structures under the influence of sun induced damage, gravity, hormonal changes in women, decreased blood supply and facial muscle activity. A thorough knowledge of these changes helps the facial plastic surgeon to choose the best protocol of rejuvenating the aging face by a combination of a variety of face lift operations, thread-lift injection of fat or fillers, botulinum toxin, lasers and mesotherapy to get an optimum result [Figures 10 and 11].

\section{SUGGESTED READING}

1. Aiache $A$, Ramirez $O$. The suborbicularis oculi fat pads: Anatomic and clinical study. Plast Reconstr Surg 1995;95:37-42.

2. Baker DC, Conley J. Avoiding facial nerve injuries in rhytidectomy. Plast Reconstr Surg 1979;64:781-95.

3. Barton F, Gyimesi I. Anatomy of the nasolabial fold. Plast Reconstr Surg 1997;100:1276-80.

4. Furnas D. The retaining ligaments of the face. Plast Reconstr Surg 1989;83:11-6.

5. Fisher G, Wang Z. Pathophysiology of premature skin aging induced by ultraviolet light. N Engl J Med 1997;13;337:1419-28.

6. Guinot C, Malvy DJ, Ambroisine L, Latreille J, Mauger E, Tenenhaus M, et al. Relative contribution of Intrinsic vs Extrinsic factors to skin aging as determined by a validated skin age score. Arch Dermatol 2002;138:1454-60.

7. HendiA, Brodland DG. Melanocytes in long-standing sun-exposed skin: Quantitative analysis using the MART-1 immunostain. Arch Dermatol 2006;142:871-6.

8. Knize D. An anatomically based study of the mechanism of eyebrow ptosis. Plast Reconstr Surg 1996;97:1321-33.

9. LaTrenta $\mathrm{G}$. The aesthetic anatomy of the face. Atlas of aesthetic face and neck surgery. WB Saunders: Philadelphia; 2004. p. 2 44.

10. LaTrenta G. The aging face. Atlas of aesthetic face and neck surgery. WB Saunders: Philadelphia; 2004. p. 46-66.

11. Lemperle G, Holmes RE, Cohen SR, Lemperle S. A classification of facial wrinkles. Plast Reconstr Surg 2001;108:1735-50.

12. Mitz V, Peyronie M. The superficial musculoaponeurotic system (SMAS) in the parotid and cheek area. Plast Reconstr Surg 1976;58:80-8.

13. Pessa JE, Garza PA, Love VM, Zadoo VP, Garza JR. The anatomy of the labiomandibular fold. Plast Reconstr Surg 1998;101:482-6.

14. Pessa JE. An algorithm of facial aging. Plast Reconstr Surg 2000;106:479-88.

15. Rubin LR. The anatomy of the nasolabial fold: The keystone of the smiling mechanism. Plast Reconstr Surg 1999;103:687-91.

16. Stuzin JM, Baker TJ, Gordon HL. The relationship of the superficial and deep facial fascias: Relevance to rhytidectomy and aging. Plast Reconstr Surg 1992;89:441-9

17. Stuzin JM, Wagstrom L, Kawamoto HK, Wolfe SA. Anatomy of the frontal branch of the facial nerve: The significance of the temporal fat pad. Plast Reconstr Surg 1989;83:265-71.

18. Stuzin J, Baker T. Aging face and neck. In: Mathes SJ, editor. Plastic surgery. Vol 2, WB Saunders: Philadelphia; 2006. p. 159214.

19. Stuzin J, Baker T, Gordon H. The relationship of the superficial and deep facial fascias. Relevance to rhytidectomy and aging. Plast Reconstr Surg 1992;89:441-9.

20. Trautinger F. Mechanisms of photodamage of the skin and its functional consequences for skin ageing. Clin Exp Dermatol 2001;26:573-7.

21. Yousif NJ, Gosain A, Matloub HS, Sanger JR, Madiedo G, Larson DL. The nasolabial fold: An anatomic and histologic reappraisal. Plast Reconstr Surg 1994;93:60-9.

Source of Support: Nil, Conflict of Interest: None declared. 